\title{
Efficient Texture Image Retrieval of Improved Completed Robust Local Binary Pattern
}

\author{
Arrie Kurniawardhani \\ Department of Informatics \\ Universitas Islam Indonesia \\ Yogyakarta, Indonesia \\ e-mail :arrie.kurniawardhani@uii.ac.id
}

\author{
Agus Eko Minarno \\ Department of Informatics \\ UniversitasMuhammadiyah Malang \\ Malang, Indonesia \\ e-mail :agoes.minarno@gmail.com
}

\author{
Fitri Bimantoro \\ Department of Informatics \\ UniversitasMataram \\ Mataram, Indonesia
}

\begin{abstract}
Improved Completed Robust Local Binary Pattern is one of the robust texture extraction for image retrieval that rotation invariant (ICRLBP). ICRLBP has proven that can increase the precision, recall, and computation time from its previous work by $21.14 \%, 20.03 \%$, and 56 times, respectively, on four different texture image dataset. ICRLBP, however, has a lot of feature, thus require more time during recognition process. Moreover, it leads to high time consuming and curse of dimensionality. To overcome those issues, in this paper, we try to reduce insignificant or unnecessary ICRLBP attributes and examine the effect of reducing number of attributes on precision and recall of the retrieving images. The methods we used to reduce the ICRLBP attributes are Correlation-based Feature Selection (CFS) and Pearson's-basedCorrelation.The experiment results show that those feature selections not only can reduce number of attributes but also can improve precision, recall, and computation time. For S_M_C feature (sign, magnitude, and center features of ICRLBP are ploted on histogram jointly), CFS can reduce up to $95 \%$ number of attributes and improve precision, recall, computation time up to $7.5 \%, 7.1 \%, 11.42$ times, respectively. For $M_{-} C$ feature (magnitude and center features of ICRLBP are ploted on histogram jointly), CFS can reduce up to $4.2 \%$ number of attributes and improve precision, recall, computation time up to $4.2 \%, 4 \%, 1.1$ times, respectively. It indicated that Correlation-based Feature Selection (CFS) and Pearson's-basedCorrelation can reduce the ICRLBP attributes effectively.
\end{abstract}

Keywords-Attribute Selection, Batik, Content Based Image Retrieval, Correlation-based Feature Selection, Improved Completed Robust Local Binar.

\section{INTRODUCTION}

Since 1990s, the amounts of researches have been conducted for content-based image retrieval (CBIR) [1]. It attracts much attention because images became the most important media format for communication which they contain a rich amount of information. Image retrieval to retrieve texture image still have big challenge to achieve high retrieval accuracy and less computational complexity.

A number of studies have been conducted to retrieve texture image precisely. Kokare, Biswas, andChatterji did texture image retrieval using rotated wavelet filtersbased approach to improves the retrieval rate of traditional discrete wavelet transform [2][3]. Reference [4][5] did texture image retrieval using Gabor feature based approach. Reference $[6][7][8][9][10]$ did image retrieval using texton or texture of element based approach that compiled of texture, color, and shape feature extraction simultaneously. Reference [11] did image retrieval using Improved Completed Robust Local Binary Pattern (ICRLBP)based approach to improves Local Binary Pattern (LBP) in order to rotation invariant and insensitive to noise.

The performance of ICRLBP was evaluated by retrieving 18 images that have different rotation angle based on a query image. ICRLBP has proven that it can improve the previous work, Completed Robust Local Binary Pattern (CRLBP). ICRLBP increased precision, recall, and computation time by $21.14 \%, 20.03 \%$, and 56 times, respectively, on four different texture image dataset. The optimal precision and recall of ICRLBP can achieve are $84.86 \%$ and $80.39 \%$, respectively, using $\mathrm{S} \mathrm{M} \mathrm{C}$ feature(sign, magnitude, and center features of ICRLBP $\overline{\text { are }}$ ploted on histogram jointly). S_M_C feature of ICRLBP, however, has a lot of feature, i.e. 2592 attributes, thus require more time during recognition process. Moreover, it leads to high time consuming and curse of dimensionality.

To overcome those issues, in this paper, we try to reduce insignificant or unnecessary ICRLBP attributes and examine the effect of reducing number of attributes on precision and recall of the retrieving images. The methods we used to reduce the ICRLBP attributes are Correlation-based Feature Selection (CFS) [12][13] and Pearson's-basedCorrelation [14]. 


\section{Previous Work}

\section{A. Improved Completed Robust Local Binary Pattern}

This section will explain a brief review previous work, Improved Completed Robust Local Binary Pattern [11][15].Improved Completed Robust Local Binary Pattern (ICRLBP) is proposed to improve the performance of Completed Robust Local Binary Pattern (CRLBP)[16] in order to be rotation invariant. ICRLBP improves the performance of CRLBP by inserting LBPROTalgorithm [17] to CRLBP algorithm.

ICRLBP represents the texture locally based on local difference sign intensity of neighbor pixels to the threshold value (CRLBP_S), magnitude intensity of neighbor pixels to the threshold value (CRLBP_M), and center pixel intensity (CRLBP_C), as defined in (1), (4), and (5), respectively.

$$
\begin{gathered}
C R L B P \_S=\sum_{p=0}^{P-1} s\left(I_{p, R}-W L G_{C}\right) 2^{p} \\
=\sum_{p=0}^{P-1} S\left(I_{p, R}-\frac{\sum_{i=0}^{P-1} I_{c i, R}+\alpha I_{C}}{P+\alpha}\right)
\end{gathered}
$$

where, Weighted Local Gray Level (WLG) is a threshold value as defined in (2) and $s(x)$ is defined in (3)

$$
\begin{array}{r}
W L G=\frac{\sum_{p=0}^{P-1} I_{p, R}+\alpha I_{C}}{P+\alpha} \\
s(x)= \begin{cases}1, & x \geq 0 \\
0, & x<0\end{cases}
\end{array}
$$

$P$ is the total number of involved neighbor pixels, $R$ is radius between center pixel and neighbor pixels, $I_{c}$ is value of center pixel intensity, $I_{p, R}$ is value of $p^{\text {th }}$ involved neighbor pixel intensity $(p=0,1, \ldots, P-1)$ with radius $R, \alpha$ is a parameter set by user, and $I_{c i, R}$ is value of $i^{\text {th }}$ involved neighbor pixel intensity $(i=0,1, \ldots, P-1)$ with radius $R$ from center pixel $I_{c}$.

$$
C R L B P_{-} M=\sum_{p=0}^{P-1} s\left(m_{p}-c\right) 2^{p}
$$

where,

$$
\begin{aligned}
& m_{p}=\left|W L G_{p}-W L G_{c}\right| \\
& =\left|\frac{\sum_{i=0}^{P-1} I_{p i, R}+\alpha I_{p, R}}{P+\alpha}-\frac{\sum_{i=0}^{P-1} I_{c i, R}+\alpha I_{c}}{P+\alpha}\right|
\end{aligned}
$$

$I_{p i, R}$ is value of $i^{\text {th }}$ involved neighbor pixel intensity ( $i=$ $0,1, \ldots, P-1$ ) with radius $R$ from center pixel $I_{p, R}$. Where as $c$ is threshold value calculated of mean value of $m_{p}$ of a whole image. CRLBP_M is calculated the local variance of WLG.

CRLBP_Coperator represents value of center pixel intensity as defined in (5).

$$
C R L B P_{-} C=s\left(W L G_{c}-c_{I}\right)
$$

Where $c_{I}$ is threshold calculated of mean value of Average Local Gray Level (ALG) of a whole image. ALG is defined in Eq. (6).

$$
A L G=\frac{\sum_{p=0}^{P-1} I_{p, R}+I_{c}}{P}
$$

After the value of CRLBP_S,CRLBP_S, and CRLBP_S are obtained, LBPROT algorithm is inserted after binary value of sign vector and magnitude vector is obtained. LBPROT looks for the smallest combination of binary value of sign vector and magnitude vector in each pixel, as defined in(7). Where $C R L B P_{P, R}$ is the binary value obtained from texture feature extraction of CRLBP, and $P$ is the total number of shifted combination that has the same total number of involved neighbor pixels.

$$
\operatorname{LBPROT}_{P, R}=\min \left\{\operatorname{ROT}\left(\operatorname{CRLBP} P_{P, R}, p\right)\right\}
$$

The smallest combination of binary value is converted to decimal value. From the decimal value, feature histogram of ICRLBP_Sign ICRLBP Magnitude(ICRLBP M), and ICRLBP Center (ICRLBP_C) can be plotted.

In grayscale image, if the value of $P$ is 8 , then histogram of $C R L B P \_S, C R L B P \_M$, and $C R L B P \_C$ consist of 36,36 , and 2 bin features, respectively. Those histograms can be combined together to get the final histogram of ICRLBP. Based on Guo [18], there are two ways to combined those histograms, namely concatenate and jointly.

\section{B. Dataset}

Four image datasets are used for evaluating the performance of ICRLBP, namely Batik, textile, Brodatz, and Corel. The size of Batik, textile, and Brodatz image is $128 \times 128$ pixels, whereas the size of Corel image is $80 \times 80$ pixels. All images are in JPEG format. Batik and Brodatz dataset consist of 112 types of image or class, whereas textile and Corel dataset consist of 50 types of image or class. Each types of those images is rotated by $0^{\circ}, 5^{\circ}, 10^{\circ}, 15^{\circ}, 20^{\circ}, 25^{\circ}$, $30^{\circ}, 35^{\circ}, 40^{\circ}, 45^{\circ}, 50^{\circ}, 55^{\circ}, 60^{\circ}, 65^{\circ}, 70^{\circ}, 75^{\circ}, 80^{\circ}, 85^{\circ}, 90^{\circ}$. Therefore, There are 19 images in each class. The example of images in one class as shown in Fig.1.

Batik is the traditional pattern on a fabric drawn with traditional method [19]. Batik has been recognized by UNESCO as one of the indigenous cultural heritage of Indonesia, on October 2, 2009. Batik and textile datasets are obtained from the Laboratory of Vision, Image Processing and Graphics (VIP-G) InstitutTeknologiSepuluhNopember. Brodatz dataset is obtained from (http://sipi.usc.edu/database/database.php?volume=textures). Corel dataset used in this study is Corel 5,000 which is obtained from (http://www.ci.gxnu.edu.cn/cbir/Dataset.aspx). Corel 5,000 consists of 50 categories, and each category consists of 100 images. But in this study, just 1 image of each category is used that is chosen randomly. Batik, textile, 
Brodatz, and Corel datasets are showed in Fig. 2-5 respectively.
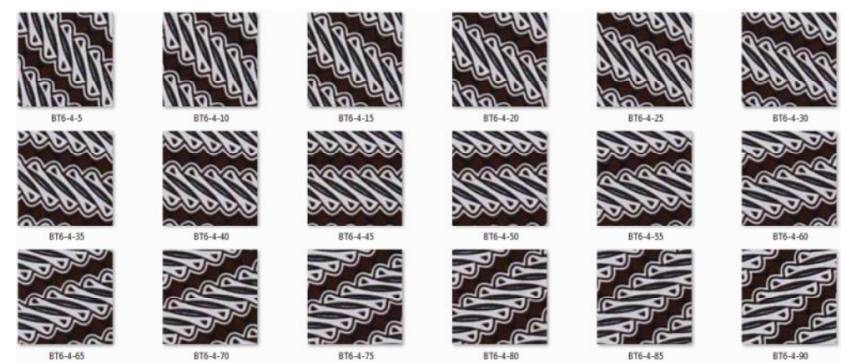

Fig. 1. Example of images in one class of Batik dataset.
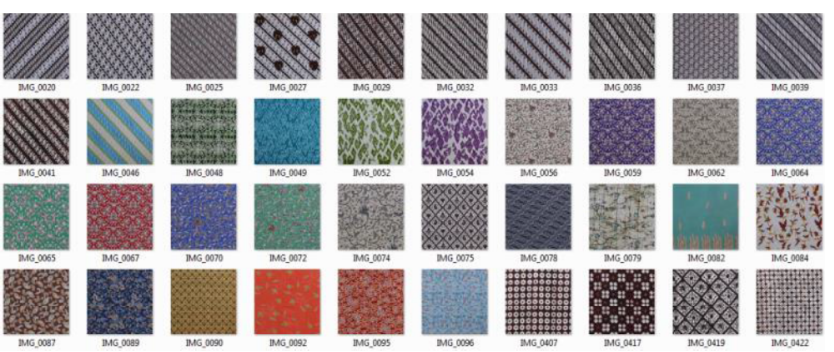

Fig. 2. Example of Batik dataset.
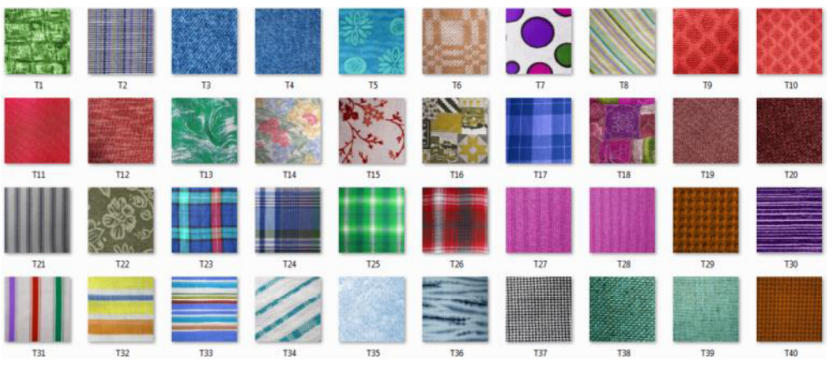

Fig. 3. Example of Textile dataset.
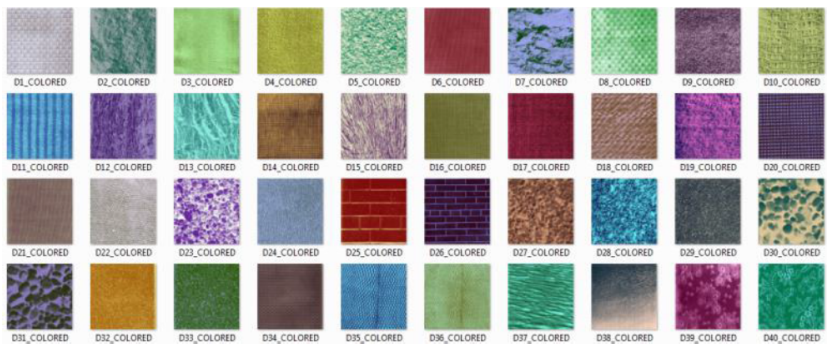

Fig. 4. Example of Brodatz dataset.

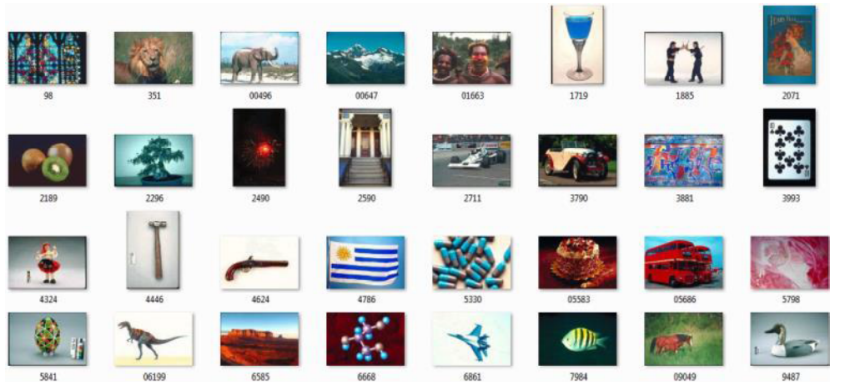

Fig. 5. Example of Corel dataset.

\section{Methodology}

In this study, we use the same dataset and algorithm as in [11]. According to the [11], the feature combinations that can achieve the optimal precision and recall are $\mathrm{M} \mathrm{C}$ featurewhen using Modified Canberra distance and S_M_C featurewhen using L1 distance, as defined in Table I. Because of that, the ICRLBP features that we observe in this study are only M_C feature andS_M_C feature.

As previously mentioned in [11], M_C feature of ICRLBP has 72 attributes that we need to reduce, whereas S_M_C feature has 2592 attributes. We reduce those attributes after we generate them and before we fed them to distance measure. Attribute selection methods we used are Correlation-based Feature Selection (CFS) [12][13]. and Pearson'sbasedCorrelation [14].

\section{A. Correlation-based Feature Selection \\ Define.}

Correlation-based Feature Selection (CFS) produces a subset of attributes or reduced attributes without losing the essential signal in the data [13]. CFS identifies attributes that are strongly associated to the target class and weakly associated with each other attribute. CFS evaluates the worth of a subset of attributes by considering the individual predictive ability of each feature along with the degree of redundancy between them. Subsets of features that are highly correlated with the class while having low intercorrelation are preferred.

\section{B. Pearson's-based Correlation}

Pearson's-based Correlationevaluates the worth of an attribute by measuring the correlation based on Pearson's between it and the class.

\section{Distance Measure}

The similarity between query images and images in the database is measured using L1 and modified Canberra distance [9], that are defined in equation (8) and (9), respectively.

$$
\begin{gathered}
L D(T, Q)=\sum_{i=1}^{F}|I i-Q i| \\
C D(T, Q)=\sum_{i=1}^{F} \frac{|I i-Q i|}{|I i+\mu I|+|Q i+\mu Q|}
\end{gathered}
$$


Where I is image in the dataset, $\mathrm{Q}$ is query image, $\mathrm{F}$ is the number of feature vector of each image, $\mu I=$ $\sum_{i=1}^{F} \frac{I i}{F}$ and $\mu Q=\sum_{i=1}^{F} \frac{Q i}{F}$.

\section{Performance Measure}

The performance of ICRLBP is measured using precision and recall which are defined in equation (10) and (11), respectively.

$$
\begin{gathered}
\text { Precision }=I r / n .100 \% \\
\text { Recall }=I r / m .100 \%
\end{gathered}
$$

Where $I_{r}$ is the number of retrieved images, $n$ is the number of relevant images that must be retrieved, and $m$ is the number of all relevant data in the dataset.

\section{RESULT AND DISCUSSION}

The performance of ICRLBP is evaluated with six scenarios on the four databases. The first scenario looks for the ICRLBP precision, recall, and computation time of unreduced M C. The second scenario looks for the ICRLBP precision, recall, and computation time of unreduced S_M_C feature. The third scenario looks for the ICRLBP precision, recall, and computation time of reduced $\mathrm{M} C$ featureusing CFS. The fourth scenario looks for the ICRLBP precision, recall, and computation time of unreduced $\mathrm{S}$ M C featureusing CFS. The fifth scenario looks for the ICR $\bar{B} \bar{B} P$ precision, recall, and computation time of reduced M_C featureusing Pearson's-basedCorrelation. The sixth scenario looks for the ICRLBP precision, recall, and computation time of unreduced S_M_C featureusing Pearson's-basedCorrelation.

In each scenario, each image of each database becomes a query image alternately. The total number of image that are retrieved in each retrieval process of one query image is 18 images, because there are 18 images in one class that are rotated in different angles.

Table I show the result of first and second scenario. Table I indicate that the optimal precisionsof unreduced $\mathrm{M} \mathrm{C}$ feature that can achieve of Batik, Brodatz, Corel, and Textile dataset are $80.75 \%, 65.73 \%, 64.06 \%$, and $81.92 \%$, respectively. Whereas, the optimal recallof unreduced M_C feature that can achieve of Batik, Brodatz, Corel, and Textile dataset are $76.5 \%, 62.27 \%, 60.69 \%$, and $77.61 \%$, respectively. Those all optimal precision and recall are achieved using Modified Canberra distance. The unreduced $\mathrm{M}_{-} \mathrm{C}$ feature has 72 attributes.

Table I also indicate about the optimal precisionsof unreduced S M C feature that can achieve of Batik, Brodatz, Corel, and Textile dataset, that is $80.48 \%, 66.24 \%, 59.92 \%$, and $84.86 \%$, respectively. Whereas, the optimal recallof unreduced S M C feature that can achieve of Batik, Brodatz, Corel, and Textile dataset are $76.25 \%, 62.75 \%, 56.76 \%$, and $80.39 \%$, respectively. The unreduced S_M_C feature has 2592 attributes.

Table II show the result of thirdand fourth scenario. Table II indicate that the optimal precisionsof reduced M_C feature that can achieve of Batik, Brodatz, Corel, and Textile dataset are $84.02 \%, 69.84 \%, 68.77 \%$, and $86.8 \%$, respectively. Whereas, the optimal recallof reduced $\mathrm{M} \mathrm{C}$ feature that can achieve of Batik, Brodatz, Corel, and Textile dataset are $79.6 \%, 66.16 \%, 65.15 \%$, and $82.34 \%$, respectively. Those all optimal precision and recall are achieved using Modified Canberra distance. The reduced $\mathrm{M} \mathrm{C}$ feature using CFS has approximately 38 attributes. It is indicated that CFS can reduce the attributes of $\mathrm{MC}$ feature around $47 \%$, without reducing the value of precision and recall instead increasing precision, recall, and computation time around $4.2 \%, 4 \%$, and 1.1 times, respectively.

Table II also indicate about the optimal precisionsof unreduced S M C feature that can achieve of Batik, Brodatz, Corel, and Textile dataset, that is $87.21 \%, 74.18 \%, 71.78 \%$, and $88.2 \%$, respectively. Whereas, the optimal recallof unreduced S M C feature that can achieve of Batik, Brodatz, Corel, and Textile dataset are $82.62 \%, 70.27 \%, 68.01 \%$, and $83.56 \%$, respectively. Those all optimal precision and recall are achieved using Modified Canberra distance. The reduced S_M_C feature using CFS has approximately 124.5 attributes. It is indicated that CFS can reduce the attributes of S_M_C feature around $95 \%$, without reducing the value of precision and recall instead increasing precision, recall, and computation time around $7.5 \%, 7.1 \%$, and 11.4 times respectively.

Table III show the result of fifthand sixth scenario. Table III indicate that the optimal precisionsof reduced M_C feature that can achieve of Batik, Brodatz, Corel, and Textile dataset are $83.74 \%, 69.17 \%, 67.22 \%$, and $85.8 \%$, respectively. Whereas, the optimal recallof reduced M_C feature that can achieve of Batik, Brodatz, Corel, and Textile dataset are $79.33 \%, 65.53 \%, 63.68 \%$, and $81.29 \%$, respectively. Those all optimal precision and recall are achieved using Modified Canberra distance. The reduced M C feature using Pearson'sbasedCorrelation has approximately 40.5 attributes. It is indicated that Pearson's-basedCorrelation can reduce the attributes of M C feature around $44 \%$, without reducing the value of precision and recall instead increasing precision, recall, and computation time around $3.4 \%, 3.2 \%$, and 1.1 times, respectively.

Table III also indicate about the optimal precisionsof unreduced S M C feature that can achieve of Batik, Brodatz, Corel, and Textile dataset, that is $86.24 \%, 71.86 \%, 70.05 \%$, and $87.99 \%$, respectively. Whereas, the optimal recallof unreduced S M C feature that can achieve of Batik, Brodatz, Corel, and Textile dataset are $81.70 \%, 68.08 \%, 66.36 \%$, and $83.36 \%$, respectively. Those all optimal precision and recall are achieved using Modified Canberra distance. The reduced S_M_C feature using Pearson's-basedCorrelation has approximately 148 attributes. It is indicated that Pearson'sbasedCorrelation can reduce the attributes of S M C feature around $94 \%$, without reducing the value of precision and recall instead increasing precision, recall, and computation time around $6.2 \%, 5.8 \%$, and 9.3 times, respectively.

From Table I until Table III, we can show that the optimal distance measure to do image retrieval in this study is Modified Canberra distance. Beside, it shows that CFS is more optimal for attribute reducing than Pearson's-basedCorrelation in this study. Moreover, CFS is faster when searching the 
ICACSIS 2016

significant attributes than Pearson's-basedCorrelation's. TableIV and Fig. 6-7 shows the improvement performance using CFS that compare to unreduced ICRLBP using Modified Canberra distance.

According to the results mentioned above, CFS and Pearson's-basedCorrelation can reduce the attributes effectively. It is indicate that there are some attributes that are not significant or necessary. Some attributes become insignificant because they are irrelevant attribute, i.e. the attribute values of all data are constant e.g. zero value for all data, or they have low variance. Furthermore, some attributes become unnecessary because they are redundant attribute. Those insignificant and unnecessary attribute lead to curse of dimensionality, extend the image retrieval runtime, and sometimes deteriorate the precision and recall rate because of overfitting.

\section{CONCLUSION}

This study try to reduce insignificant or unnecessary ICRLBP attributes and examine the effect of reducing number of attributes on precision and recall of the retrieving images.
The methods we used to reduce the ICRLBP attributes are Correlation-based Feature Selection (CFS) and Pearson'sbasedCorrelation. The experiment results show that those feature selections not only can reduce number of attributes but also can improve precision, recall, and computation time. For S M C feature (sign, magnitude, and center features of ICRLBP are ploted on histogram jointly), CFS can reduce up to $95 \%$ number of attributes and improve precision, recall, computation time up to $7.5 \%, 7.1 \%, 11.42$ times, respectively. For M_C feature (magnitude and center features of ICRLBP are ploted on histogram jointly), CFS can reduce up to $4.2 \%$ number of attributes and improve precision, recall, computation time up to $4.2 \%, 4 \%, 1.1$ times, respectively. It is indicate that there are some attributes that are insignificant or unnecessary for image retrieval. Thus, those can extend the image retrieval runtime, and sometimes degraded the retrieval performance.

TABLE I. PRECISION AND RECALL OF UNREDUCED ICRLBP IN EACH DATASET

\begin{tabular}{|c|c|c|c|c|c|c|c|c|c|}
\hline \multirow{2}{*}{\multicolumn{2}{|c|}{$\begin{array}{c}\text { Dataset } \\
\text { Features }\end{array}$}} & \multicolumn{2}{|c|}{ Batik } & \multicolumn{2}{|c|}{ Brodatz } & \multicolumn{2}{|c|}{ Corel } & \multicolumn{2}{|c|}{ Textile } \\
\hline & & M_C & S_M_C & M_C & S_M_C & M_C & S_M_C & M_C & S_M_C \\
\hline \multirow{2}{*}{ L1 } & Precision & 61,86 & 79,22 & 53,19 & 66,24 & 46,31 & 59,92 & 79,77 & 84,86 \\
\hline & Recall & 58,61 & 75,05 & 50,39 & 62,75 & 43,87 & 56,76 & 75,57 & 80,39 \\
\hline \multirow{2}{*}{ Canberra } & Precision & 80,75 & 80,48 & 65,73 & 61,87 & 64,06 & 44,58 & 81,92 & 69,44 \\
\hline & Recall & 76,5 & 76,25 & 62,27 & 58,61 & 60,69 & 42,23 & 77,61 & 65,78 \\
\hline \multicolumn{2}{|c|}{ computation time (s) } & 143,2 & 2012 & 139,2 & 1950 & 29 & 343,8 & 28,87 & 345,2 \\
\hline \multicolumn{2}{|c|}{ number of attributes } & 72 & 2592 & 72 & 2592 & 72 & 2592 & 72 & 2592 \\
\hline
\end{tabular}

TABLE II. PRECISION AND RECALL OF REDUCED ICRLBP USING CFS IN EACH DATASET

\begin{tabular}{|c|c|c|c|c|c|c|c|c|c|}
\hline \multirow{2}{*}{\multicolumn{2}{|c|}{$\begin{array}{c}\text { Dataset } \\
\text { Features }\end{array}$}} & \multicolumn{2}{|c|}{ Batik } & \multicolumn{2}{|c|}{ Brodatz } & \multicolumn{2}{|c|}{ Corel } & \multicolumn{2}{|c|}{ Textile } \\
\hline & & M_C & S_M_C & M_C & S_M_C & M_C & S_M_C & M_C & S_M_C \\
\hline \multirow{2}{*}{ L1 } & Precision & 61,45 & 78,11 & 52,69 & 64,59 & 45,19 & 55,88 & 79,76 & 85,01 \\
\hline & Recall & 58,22 & 74 & 49,91 & 61,19 & 42,81 & 52,94 & 75,56 & 80,54 \\
\hline \multirow{2}{*}{ Canberra } & Precision & 84,02 & 87,21 & 69,84 & 74,18 & 68,77 & 71,78 & 86,8 & 88,2 \\
\hline & Recall & 79,6 & 82,62 & 66,16 & 70,27 & 65,15 & 68,01 & 82,23 & 83,56 \\
\hline \multicolumn{2}{|c|}{ computation time (s) } & 134,5 & 176,7 & 121,2 & 160 & 25,28 & 28,23 & 26,4 & 34,81 \\
\hline \multicolumn{2}{|c|}{ number of attributes } & 42 & 148 & 34 & 112 & 28 & 69 & 48 & 169 \\
\hline
\end{tabular}

TABLE III. PRECISION AND RECALl of REDUCED ICRLBP USING PEARSON'S-BASED CORRELATION IN EACH DATASET

\begin{tabular}{|c|c|c|c|c|c|c|c|c|c|}
\hline \multirow{2}{*}{\multicolumn{2}{|c|}{$\begin{array}{c}\text { Dataset } \\
\text { Features }\end{array}$}} & \multicolumn{2}{|c|}{ Batik } & \multicolumn{2}{|c|}{ Brodatz } & \multicolumn{2}{|c|}{ Corel } & \multicolumn{2}{|c|}{ Textile } \\
\hline & & M_C & S_M_C & M_C & S_M_C & M_C & S_M_C & M_C & S_M_C \\
\hline \multirow{4}{*}{ L1 } & Precision & 61,88 & 76,36 & 53,27 & 63,86 & 46,31 & 58,18 & 79,78 & 83,09 \\
\hline & Recall & 58,63 & 72,34 & 50,47 & 60,5 & 43,87 & 55,12 & 75,58 & 78,72 \\
\hline & computation time (s) & 135,6 & 388,4 & 132,4 & 163,8 & 29,27 & 33,62 & 28,89 & 33,29 \\
\hline & number of attributes & 68 & 149 & 61 & 150 & 68 & 146 & 67 & 147 \\
\hline \multirow{4}{*}{ Canberra } & Precision & 83,74 & 86,24 & 69,18 & 71,86 & 67,22 & 70,05 & 85,81 & 87,99 \\
\hline & Recall & 79,33 & 81,7 & 65,53 & 68,08 & 63,68 & 66,36 & 81,29 & 83,36 \\
\hline & computation time (s) & 128,8 & 388,4 & 129,1 & 162,8 & 28,62 & 34,87 & 25,76 & 33,29 \\
\hline & number of attributes & 48 & 149 & 48 & 149 & 53 & 147 & 13 & 147 \\
\hline
\end{tabular}


TABLE IV. THE AVERAGE IMPROVEMENT PERFORMANCE OF ICRLBP

\begin{tabular}{|c|c|c|c|c|c|c|c|c|}
\hline & \multicolumn{8}{|c|}{ The average improvement of } \\
\hline & \multicolumn{4}{|c|}{ M_C feature } & \multicolumn{4}{|c|}{ S_M_Cfeature } \\
\hline & precision (\%) & $\begin{array}{l}\text { recall } \\
(\%)\end{array}$ & $\begin{array}{c}\text { computation time } \\
\text { (times) }\end{array}$ & $\begin{array}{c}\text { number of } \\
\text { attributes (\%) }\end{array}$ & precision (\%) & $\begin{array}{c}\text { recall } \\
(\%)\end{array}$ & $\begin{array}{c}\text { computation time } \\
\text { (times) }\end{array}$ & $\begin{array}{c}\text { number of } \\
\text { attributes (\%) }\end{array}$ \\
\hline CFS & 4,2425 & 4,018 & 1,114 & 47 & 7,468 & 7,078 & 11,42 & 95 \\
\hline $\begin{array}{c}\text { Pearson's-based } \\
\text { Correlation }\end{array}$ & 3,371075 & 3,193 & 1,081 & 44 & 6,16 & 5,838 & 9,346 & 94 \\
\hline
\end{tabular}

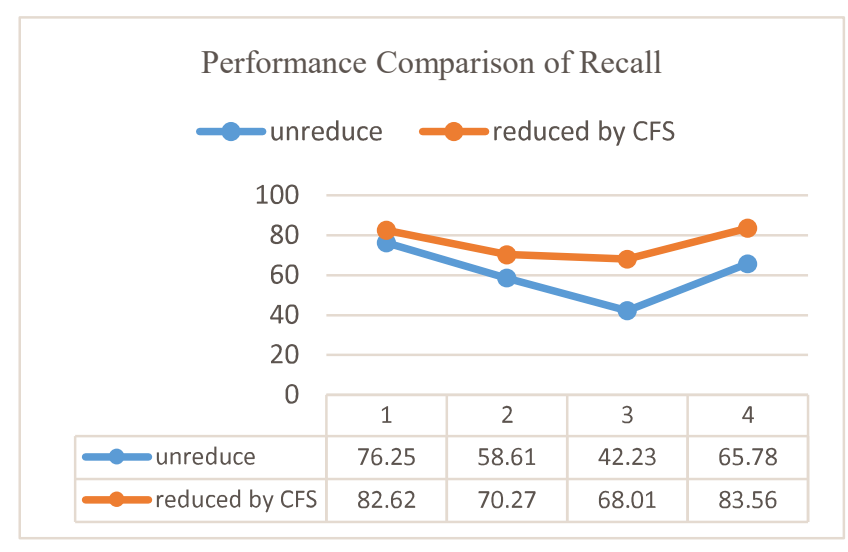

Fig. 6. Performance comparison of recall.

\section{References}

[1] Liu, Y., Zhang, D., Lu, G., \& Ma, W. Y. "A survey of content-based image retrieval with high-level semantics." Pattern recognition 40.1 (2007): 262-282.

[2] Kokare, M., Biswas, P.K. and Chatterji, B.N., 2005. Texture image retrieval using new rotated complex wavelet filters. IEEE Transactions on Systems, Man, and Cybernetics, Part B (Cybernetics), 35(6), pp.1168-1178.

[3] Kokare M, Biswas PK, Chatterji BN. Texture image retrieval using rotated wavelet filters. Pattern recognition letters. 2007 Jul 15;28(10):1240-9.

[4] Zhang D, Wong A, Indrawan M, Lu G. Content-based image retrieval using Gabor texture features. InIEEE Pacific-Rim Conference on Multimedia, University of Sydney, Australia 2000 Dec 13 (pp. 392-395).

[5] Han, J. and Ma, K.K., 2007. Rotation-invariant and scale-invariant Gabor features for texture image retrieval. Image and vision computing, 25(9), pp.1474-1481.

[6] Liu, G.H. and Yang, J.Y., 2008. Image retrieval based on the texton cooccurrence matrix. Pattern Recognition, 41(12), pp.3521-3527.

[7] Liu GH, Zhang L, Hou YK, Li ZY, Yang JY. Image retrieval based on multi-texton histogram. Pattern Recognition. 2010 Jul 31;43(7):2380-9.

[8] Liu GH, Li ZY, Zhang L, Xu Y. Image retrieval based on microstructure descriptor. Pattern Recognition. 2011 Sep 30;44(9):2123-33.

[9] Minarno, A.E., Munarko, Y., Bimantoro, F., Kurniawardhani, A. and Suciati, N., 2014, February. Batik image retrieval based on enhanced micro-structure descriptor. In Computer Aided System Engineering (APCASE), 2014 Asia-Pacific Conference on (pp. 65-70). IEEE.

[10] Minarno, A.E., Kurniawardhani, A. and Bimantoro, F., 2016. Image Retrieval Based on Multi Structure Co-occurrence Descriptor.

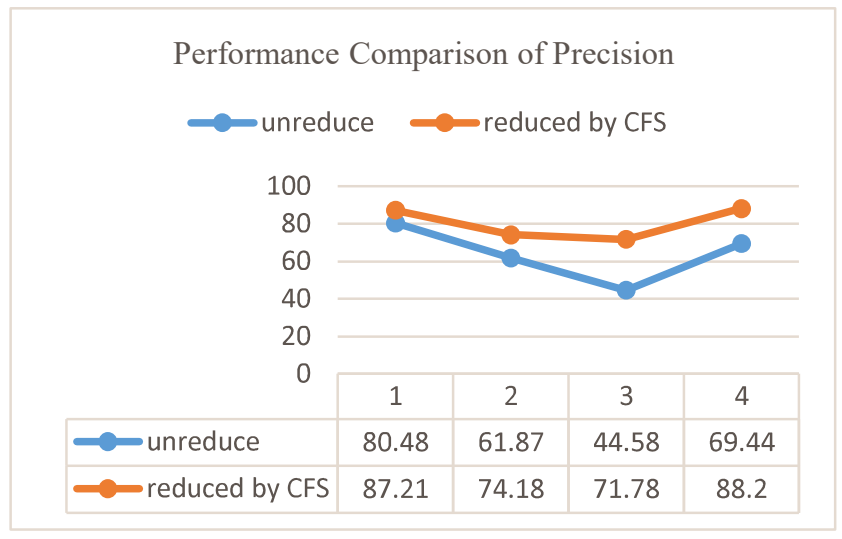

Fig.7. Performance comparison of precision.

TELKOMNIKA (Telecommunication Computing Electronics and Control), 14(3).

[11] Kurniawardhani, A., Suciati, N. and Arieshanti, I., 2015. Texture Feature Extraction Using Improved Completed Robust Local Binary Pattern for Batik Image Retrieval. International Journal of Advancements in Computing Technology, 7(6), p.69.

[12] M. A. Hall (1998). Correlation-based Feature Subset Selection for Machine Learning. Hamilton, New Zealand.

[13] Hall M, Holmes G: Benchmarking attribute selection techniques for discrete class data mining. IEEE Trans Knowl Data Eng. 2003, 15 (6): 1437-1447. 10.1109/TKDE.2003.1245283

[14] Patil, M.D. and Sane, D.S.S., 2014. Effective Classification after Dimension Reduction: A Comparative Study. International Journal of Scientific and Research Publications, 4(7), p.1.

[15] Kurniawardhani, A., Suciati, N. and Arieshanti, I., 2014. Klasifikasi Citra Batik Menggunakan Metode Ekstraksi Ciri Yang Invariant Terhadap Rotasi. JUTI: Jurnal Ilmiah Teknologi Informasi, 12(2), pp.4860.

[16] Y Zhao, W Jia, RX Hu, H Min, "Completed robust local binary pattern for texture classification", Journal of Neurocomputing (ScienceDirect), vol. 106, pp.68-76, 2013

[17] M Pietikäinen, T Ojala, Z Xu, "Rotation-invariant texture classification using feature distributions", Journal of Pattern Recognition (ScienceDirect), vol. 33, no. 1, pp.43-52, 2000.

[18] ZH Guo, L Zhang, D Zhang, "A completed modeling of local binary pattern operator for texture classification", Journal of Image Processing (IEEE), vol. 19, no. 6, pp.1657-1663, 2010.

[10] A Haake, "The role of symmetry in Javanese Batik patterns", Journal of Computers \& Mathematics with Applications (ScienceDirect), vol. 17, no.4-6, pp.815-826, 1989. 